\title{
On Bernstein Polynomials Method to the System of Abel Integral Equations
}

\author{
A. Jafarian, ${ }^{1}$ S. Measoomy Nia, ${ }^{1}$ Alireza K. Golmankhaneh, ${ }^{2}$ and D. Baleanu, ${ }^{3,4,5}$ \\ ${ }^{1}$ Department of Mathematics, Islamic Azad University, Urmia Branch, P.O. BOX 969, Urmia, Iran \\ ${ }^{2}$ Department of Physics, Islamic Azad University, Urmia Branch, P.O. BOX 969, Urmia, Iran \\ ${ }^{3}$ Department of Chemical and Materials Engineering, Faculty of Engineering, King Abdulaziz University, \\ P.O. Box 80204, Jeddah 21589, Saudi Arabia \\ ${ }^{4}$ Department of Mathematics and Computer Science, Çankaya University, 06530 Ankara, Turkey \\ ${ }^{5}$ Institute of Space Sciences, P.O. Box MG-23, 76900 Magurele-Bucharest, Romania \\ Correspondence should be addressed to Alireza K. Golmankhaneh; alirezakhalili2002@yahoo.co.in
}

Received 20 November 2013; Revised 3 March 2014; Accepted 4 March 2014; Published 7 May 2014

Academic Editor: Hossein Jafari

Copyright @ 2014 A. Jafarian et al. This is an open access article distributed under the Creative Commons Attribution License, which permits unrestricted use, distribution, and reproduction in any medium, provided the original work is properly cited.

\begin{abstract}
This paper deals with a new implementation of the Bernstein polynomials method to the numerical solution of a special kind of singular system. For this aim, first the truncated Bernstein series polynomials of the solution functions are substituted in the given problem. Using some properties of these polynomials, the solution of the problem is reduced to solve a linear system of algebraic equations. In order to confirm the reliability and accuracy of the proposed method, some weakly Abel integral equations systems with comparisons are solved in detail as numerical examples.
\end{abstract}

\section{Introduction}

Many physical, biological, or engineering processes involve relating rates of change of various quantities according to physical or other principles. In some occasions, mathematical representation of these processes appears in a natural way in terms of singular integral equations. It should be noted that these types of equations contain much more mathematical information about the applied scientific problem aroused from natural phenomena. On the other hand, much singular integral equations are not easy to solve analytically. Hence, numerical methods based on solving these equations can be unbelievably powerful. Among the numerous numerical approaches that have been offered, let us mention some well-known methods. For example, Khan and Gondal [1] merged the Laplace transform and decomposition method and then presented a new mechanism for the solution of Abel type singular integral equations, that is to say, the twostep Laplace decomposition algorithm. Bougoffa et al. [2] used the Adomian decomposition method for solving linear and nonlinear Abel integral equations. Abdulkawi et al. [3] used the Chebyshev polynomials of the second kind for the numerical solution of the Cauchy type singular integral equations of the first kind, over a finite segment. The solution of a singular integral equation with logarithmic kernel in two disjoint intervals was obtained using function theoretic method by Banerjea and Dutta in [4]. The two interval conditions yielded to the two simple simultaneous algebraic equations in two unknowns, which were solved to recover the reflection and transmission coefficients in the scattering problem. Helsing in [5] presented a fast and stable solver based on the reduction, Nystrom discretization, composite quadrature, recursive compressed inverse preconditioning, and multipole acceleration techniques for the numerical solution of these integral equations on piecewise smooth curves. Maleknejad and Salimi Shamloo [6] utilized Laplace transform of the problem and operational matrices of piecewise constant orthogonal functions to solve singular Volterra integral equations. Moreover, Huang et al. [7] offered a stable approximate inversion of Abel integral equation by using 
the Taylor expansion method. This work transformed the equation approximately to a system of linear algebraic equations for the unknown function together with its derivatives. Akel and Hussein [8] proposed a convenient and efficient numerical method for the treatment of singular integral equations of the first and second kind. The method yielded a simple and closed form of the approximate solution by means of Sinc functions with smoothing transformations. Recently, we employed a structure of artificial neural networks for approximating solution of a fuzzy type Abel integral equation. Also, the application of Legendre wavelets as a basis function is offered for solving a special kind of singular system in [9]. Recently, [10-17], wavelets method has been employed for solving a class of system of nonlinear singular fractional Volterra integrodifferential equations. In this work, shifted Chebyshev polynomials and their properties were employed to derive a general procedure for forming the operational matrix of fractional derivative for Chebyshev wavelets.

In many problems in science and engineering, we have some unknown functions which are too complicated to be determined. The Bernstein polynomials method is one of the earliest analytic-numeric algorithms for approximating the unknowns in different kinds of mathematical problems. This is an extremely useful way of expressing a complicated function in terms of simple polynomial. The only requirement is that the given function should be smooth. In other words, at a point of interest it must be possible to differentiate the function as often as we please. In our earlier works, the linear integral equations system was handled by using these kinds of polynomials [18]. Moreover, in [19-22] Bernstein polynomials and Bernstein operational matrices were employed for solving some types of singular integral and integrodifferential equations. In this paper, we are going to apply a development of the Bernstein series method to approximate solution of a system of weakly Abel integral equations which has been considered in the standard form:

$$
\begin{array}{r}
\sum_{j=1}^{m} g_{i, j}(s) \varphi_{j}(s)=f_{i}(s)+\sum_{j=1}^{m} \lambda_{i, j} \int_{0}^{s} \frac{\varphi_{j}(t)}{\sqrt{s-t}} d t \\
0<s \leq 1, \quad i=1, \ldots, m,
\end{array}
$$

where $\lambda_{i, j}$ is a real constant, $f_{i}(s)$ and $g_{i, j}(s)$ are predetermined real-valued functions, and $\varphi(s)=\left[\varphi_{1}(s), \ldots, \varphi_{m}(s)\right]$ is the vector solution that will be determined. For more details on the integral equations, the reader is referred to the book [23]. Supposedly the present problem has a unique solution [23], a comfort computational form of the above system is obtained when the Bernstein polynomials are used as basis functions. Now, putting $s=s_{i}$ (for $i=0, \ldots, n$ ) transforms the resulting system to a system of linear algebraic equations for the unknown functions. Then, a numerical solution can be obtained by solving the resulting system using a standard rule. If more and more terms are used from the Bernstein series, then the polynomial representations better and better approximate the unknowns. It is clear that the $n$-th order series solution converges to the exact solution if the unknown functions are polynomials of degree up to $n$. Here is an outline of the paper. Section 2 intends to describe how to find approximate solution of a weakly Abel integral equations system by using the present approach. In Section 3, the error analysis of approximate solution corresponding to the singular integral equations system is given. To demonstrate the efficiency and reliability of the method, some numerical test examples with comparisons are given in Section 4 . Section 5 concludes the paper.

\section{Description of the Method}

The Bernstein polynomials representation is extremely important from many points of view. This approach can be used to approximate more complicated functions in terms of simpler polynomials. In this section, our aim is to show how the Bernstein polynomials method can be applied to the numerical solution of singular system (1). First, assume that the functions $f_{i}(s)$ and $g_{i, j}(s)$ are continually differentiable in the interval of interest. We know that the Bernstein approximation of function $\varphi_{j}:[0,1] \rightarrow \mathbb{R}$ is the polynomial:

$$
\varphi_{j, n}(s)=\sum_{p=0}^{n} \xi_{j, p} \beta_{p, n}(s),
$$

where $\xi_{p}$ is a constant coefficient and $\beta_{p, n}(s)$ is the Bernstein basis polynomial of degree $n$ for each positive integer $n$, which is defined by Mandal and Bhattacharya [24]:

$$
\beta_{p, n}(s)=\left(\begin{array}{l}
n \\
p
\end{array}\right) s^{p}(1-s)^{n-p}, \quad p=0, \ldots, n .
$$

It is clear that the value of $\varphi_{j, n}(s)$ approaches $\varphi_{j}(s)$ as $n$ gets larger. Suppose we carry this argument and try to determine polynomial approximations of degree $n$ for the solutions in the given problem. To do this, first $\varphi_{j, n}$ is substituted for $\varphi_{j}$ in system (1). Now, we get

$$
\begin{aligned}
& \sum_{j=1}^{m} \sum_{k=0}^{n} g_{i, j}(s) \beta_{k, n}(s) \xi_{j, k} \\
& \quad=f_{i}(s)+\sum_{j=1}^{m} \sum_{k=0}^{n} \lambda_{i, j} \int_{0}^{s} \frac{\beta_{k, n}(t)}{\sqrt{s-t}} d t \xi_{j, k},
\end{aligned}
$$

or equivalently

$$
\begin{aligned}
& \sum_{j=1}^{m} \sum_{k=0}^{n} g_{i, j}(s) \beta_{k, n}(s) \xi_{j, k} \\
& \quad=f_{i}(s)+\sum_{j=1}^{m} \sum_{k=0}^{n} \lambda_{i, j}\left(\begin{array}{l}
n \\
k
\end{array}\right) \int_{a}^{s} \frac{t^{k} \zeta_{k}(t)}{\sqrt{s-t}} d t \xi_{j, k},
\end{aligned}
$$

where

$$
\zeta_{k}(t)=\sum_{q=0}^{n-k}\left(\begin{array}{c}
n-k \\
q
\end{array}\right)(-1)^{n-k-q} t^{n-k-q} .
$$


After some simplifications, (5) is reduced to the following form:

$$
\begin{aligned}
& \sum_{j=1}^{m} \sum_{k=0}^{n} g_{i, j}(s) \beta_{k, n}(s) \xi_{j, k} \\
& \quad=f_{i}(s)+\sum_{j=1}^{m} \sum_{k=0}^{n} \lambda_{i, j}\left(\begin{array}{l}
n \\
k
\end{array}\right) \sum_{q=0}^{n-k} d_{k, q} \mu_{n-q}(s) \xi_{j, k},
\end{aligned}
$$

where

$$
\begin{aligned}
d_{k, q} & =\left(\begin{array}{c}
n-k \\
q
\end{array}\right)(-1)^{n-k-q}, \\
\mu_{n-q}(s) & =\frac{2^{n-q+1} \Gamma(n-q+1) s^{n-q+(1 / 2)}}{1 \cdot 3 \cdot 5 \cdots(2(n-q)+1)} .
\end{aligned}
$$

For suitable choice of the distinct values $s=s_{p}$ (for $p=$ $0, \ldots, n)$ in $[0,1]\left(\right.$ e.g., $\left.s_{p}=(p / n)\right)$, we achieve the following linear algebraic system:

$$
\sum_{j=1}^{m} \sum_{k=0}^{n} \gamma_{i, j}^{p, k} \cdot \xi_{j, k}=f_{i}\left(s_{p}\right), \quad i=1, \ldots, m ; p=0, \ldots, n,
$$

where

$$
\gamma_{i, j}^{p, k}=\frac{\left(\begin{array}{l}
n \\
k
\end{array}\right) n^{n}}{p^{k}(n-p)^{n-k}} g_{i, j}\left(s_{p}\right)-\lambda_{i, j}\left(\begin{array}{l}
n \\
k
\end{array}\right) \sum_{q=0}^{n-k} d_{k, q} \mu_{n-q}\left(s_{p}\right) .
$$

Therefore, system (9) with $m(n+1)$ linear equations of $m(n+$ 1) unknowns $\varphi_{j}\left(s_{p}\right)$ (for $\left.p=0, \ldots, n ; j=1, \ldots, m\right)$ can be rewritten in the matrix form as follows:

$$
\left[\begin{array}{ccc}
\Upsilon_{1,1} & \cdots & \Upsilon_{1, m} \\
\vdots & \ddots & \vdots \\
\Upsilon_{m, 1} & \cdots & \Upsilon_{m, m}
\end{array}\right]\left[\begin{array}{c}
\Phi_{1} \\
\vdots \\
\Phi_{m}
\end{array}\right]=\left[\begin{array}{c}
F_{1} \\
\vdots \\
F_{m}
\end{array}\right]
$$

or equivalently

$$
\Upsilon^{n, n} \Phi^{n}=\digamma^{n}
$$

with the block matrices:

$$
\begin{gathered}
\Upsilon_{i, j}=\left[\begin{array}{ccc}
\gamma_{i, j}^{0,0} & \cdots & \gamma_{i, j}^{0, n} \\
\vdots & \ddots & \vdots \\
\gamma_{i, j}^{n, 0} & \cdots & \gamma_{i, j}^{n, n}
\end{array}\right], \quad \Phi_{j}=\left[\begin{array}{c}
\xi_{j, 0} \\
\vdots \\
\xi_{j, n}
\end{array}\right], \\
F_{i}=\left[\begin{array}{c}
f_{i}\left(s_{0}\right) \\
\vdots \\
f_{i}\left(s_{n}\right)
\end{array}\right] .
\end{gathered}
$$

Consequently, system (11) can be solved using a standard rule to obtain the unknown constants $\xi_{j, k}$ (for $j=1, \ldots, m ; k=$ $0, \ldots, n)$. Ultimately, the unknown function $\varphi_{j}(s)$ can be easily approximated using the aforementioned assumptions.

\section{Convergence Analysis}

In this section, we intend to prove that the presented numerical method converges to the exact solution of system (1).

Theorem 1. Let $\varphi_{j, n}(s)$ (for $j=1, \ldots, m$ ) be polynomials of degree $n$ that their numerical coefficients are produced by solving the linear system (11). There exists an integer $N$ such that, for all $n \geq N$, these polynomials converge to the exact solution of the integral equations system (1).

Proof. We know that the Bernstein polynomials for the function $\varphi_{j}(s)$ that is continuous on interval $[0,1]$ converge uniformly to this function [24]. Consider again system (1). In other words, $\varphi_{j}(s)$ can be expanded as a uniformly convergent Bernstein series in $[0,1]$ :

$$
\varphi_{j}(s)=\sum_{p=0}^{n} \xi_{j, p} \beta_{p, n}(s), \quad n \geq N .
$$

Based on the suggested process, Abel integral equations system (1) can be transformed to the following equivalent infinitely systems of linear equations for unknown $\varphi_{j}(s)$ :

$$
\Upsilon \Phi=F,
$$

with

$$
\Upsilon=\lim _{n \rightarrow \infty} Y^{n, n}, \quad F=\lim _{n \rightarrow \infty} F^{n}, \quad \Phi=\lim _{n \rightarrow \infty} \Phi^{n},
$$

where $Y^{n, n}, F^{n}$, and $F^{n}$ were defined in the previous section. For the above system, the unique solution can be expressed as

$$
\Phi=L_{\digamma},
$$

where $L=\Upsilon^{-1}$. Alternatively, the above system can be rewritten as

$$
\left[\begin{array}{c}
\Phi^{n} \\
\Phi^{\infty}
\end{array}\right]=\left[\begin{array}{cc}
L^{n, n} & L^{n, \infty} \\
L^{\infty, n} & L^{\infty, \infty}
\end{array}\right]\left[\begin{array}{c}
F^{n} \\
F^{\infty}
\end{array}\right]
$$

where

$$
L^{n, n}=\left[\begin{array}{ccc}
L_{1,1}^{n, n} & \cdots & L_{1, m}^{n, n} \\
\vdots & \ddots & \vdots \\
L_{m, 1}^{n, n} & \cdots & L_{m, m}^{n, n}
\end{array}\right]
$$

Consequently, one finds that the vector $\varphi_{j, n}$ composed of the first $n+1$ elements of the exact solution vector $\varphi_{j}$ must satisfy the following relation:

$$
\Phi^{n}=L^{n, n} F^{n}+L^{n, \infty} F^{\infty} .
$$

In addition, based on the analysis of the foregoing section, the unique solution of (11) is denoted as

$$
\bar{\Phi}^{n}=\left(Y^{n, n}\right)^{-1} F^{n} .
$$


Subtracting (21) from (20) yields

$$
\Phi^{n}-\bar{\Phi}^{n}=A^{n, n} F^{n}+L^{n, \infty} F^{\infty}
$$

where $A^{n, n}=L^{n, n}-\left(\Upsilon^{n, n}\right)^{-1}$. Expanding the right-hand side of (22), the left-hand side of this is expressed by

$$
\begin{aligned}
\varphi_{i}(s)-\varphi_{i, n}(s)= & \sum_{q=0}^{n} \sum_{r=0}^{n} a_{i, j}^{q, r} f_{i}\left(s_{r}\right) \beta_{q, n}(s) \\
& +\sum_{q=0}^{n} \sum_{r=n+1}^{\infty} l_{i, j}^{q, r} f_{i}\left(s_{r}\right) \beta_{q, n}(s),
\end{aligned}
$$

where $a_{i, j}^{q, r}$ and $l_{i, j}^{q, r}$ are the elements of $A_{i, j}$ and $L_{i, j}$, respectively. Thus,

$$
\begin{aligned}
\mid \varphi_{i}(s) & -\varphi_{i, n}(s) \mid \\
\leq & \left(\left(\sum_{q=0}^{n} \sum_{r=0}^{n}\left|a_{i, j}\right|^{2}\right)^{1 / 2}-\left(\sum_{q=0}^{n} \sum_{r=n+1}^{\infty}\left|l_{i, j}\right|^{2}\right)^{1 / 2}\right), \\
& \left(\sum_{q=0}^{n} \sum_{r=0}^{n}\left|f_{i}\left(s_{r}\right)\right|^{2}\right)^{1 / 2}\left(\sum_{q=0}^{n} \sum_{r=0}^{n}\left|\beta_{q, n}(s)\right|^{2}\right)^{1 / 2}
\end{aligned}
$$

follows from the well-known Cauchy-Schwarz inequality. Since $\lim _{n \rightarrow \infty} A_{n, n}=0$ and $\lim _{n \rightarrow \infty} L_{n, \infty}=0$, so we can conclude that $\lim _{n \rightarrow \infty}\left|\varphi_{i}(s)-\varphi_{i, n}(s)\right|=0$ and proof is completed.

\section{Numerical Examples}

In this section, the above-mentioned method is illustrated with the help of three illustrative examples which include second kind Volterra integral equations system with Abel kernels. Note that the empirical results obtained here will be compared with the ones achieved from the Taylor expansion method (TEM) of degree $n$ [7].

Example 1. Consider the following singular integral equations system:

$$
\begin{aligned}
& s^{2} \varphi_{1}(s)-(s+1) \varphi_{2}(s) \\
& =f_{1}(s)+\int_{0}^{s} \frac{\varphi_{1}(t)}{\sqrt{s-t}} d t-\int_{0}^{s} \frac{\varphi_{2}(t)}{\sqrt{s-t}} d t, \\
& s \varphi_{1}(s)+\left(s^{2}+2\right) \varphi_{2}(s) \\
& =f_{2}(s)+\int_{0}^{s} \frac{\varphi_{1}(t)}{\sqrt{s-t}} d t+\int_{0}^{s} \frac{\varphi_{2}(t)}{\sqrt{s-t}} d t, \\
& 0<s \leq 1,
\end{aligned}
$$

where

$$
\begin{aligned}
f_{1}(s)= & s\left(\frac{20 s^{1 / 2}}{3}-4\right)+s^{2}\left(\frac{16 s^{1 / 2}}{15}-4\right) \\
& -4 s^{1 / 2}-s^{4}+s^{5}-\frac{32 s^{2}}{35}+1, \\
f_{2}(s)= & s^{2}\left(\frac{16 s^{1 / 2}}{15}-1\right)-s\left(\frac{20 s^{1 / 2}}{3}-11\right) \\
& -s^{3}\left(\frac{32 s^{1 / 2}}{35}-4\right)+s^{4}-2,
\end{aligned}
$$

with the exact vector solution $\left[\varphi_{1}(s), \varphi_{2}(s)\right]=\left[s^{3}-s^{2}+1,5 s-\right.$ $1]$. In this example, we aim to apply the present technique to approximate solution of the problem by taking $n=3$. The solutions in series forms are given by

$$
\begin{aligned}
\varphi_{1,3}(s)= & 3 s(s-1)^{2}-2 s^{2}(s-1)-(s-1)^{3} \\
& +s^{3}+1=s^{3}-s^{2}+1, \\
\varphi_{2,3}(s)= & 2 s(s-1)^{2}-7 s^{2}(s-1)+(s-1)^{3}+4 s^{3} \\
= & 5 s-1 .
\end{aligned}
$$

As shown, the method is quite accurate when the solutions are polynomials of degree up to $n$.

Example 2. Consider the system of Abel integral equations:

$$
\begin{aligned}
& \varphi_{1}(s)= e^{s}\left(\frac{\operatorname{erf}(\sqrt{s})-\sqrt{\pi}}{\sqrt{\pi}}\right)-\frac{s}{2} \\
&+\int_{0}^{s} \frac{\varphi_{1}(t)+\varphi_{2}(t)}{\sqrt{s-t}} d t, \\
& \varphi_{2}(s)=(s+1)\left(\frac{1+\pi \sqrt{s}}{\pi \sqrt{s}}\right) \\
& \pm e^{s}+2+\int_{0}^{s} \frac{\varphi_{1}(t)-2 \varphi_{2}(t)}{\sqrt{s-t}} d t, \\
& 0<s \leq 1,
\end{aligned}
$$

which has the exact solutions $\varphi_{1}(s)=e^{s} \operatorname{erf}(\sqrt{s}) / \sqrt{\pi}$ and $\varphi_{2}(s)=((1+s) / \pi \sqrt{s})$. For suitable distinct points $s=s_{i}$ (for $i=0, \ldots, 5)$ in $(0,1]$ in which $s_{0}$ is taken near 0 and $s_{5}=1$, the numerical results obtained here and the Taylor expansion quadrature method are presented in Table 1. Also, the exact and approximate solutions are compared in Figure 1. 


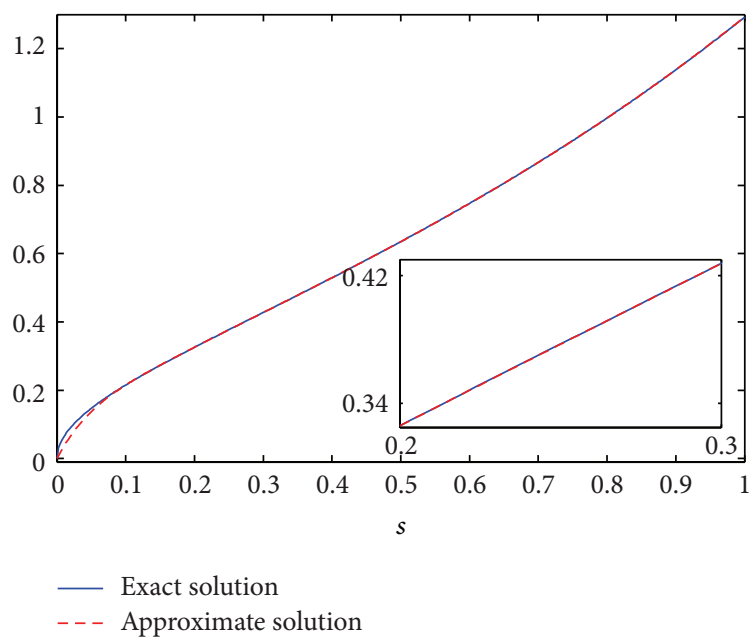

(a) $\varphi_{1}(s)$ and $\varphi_{1,5}(s)$

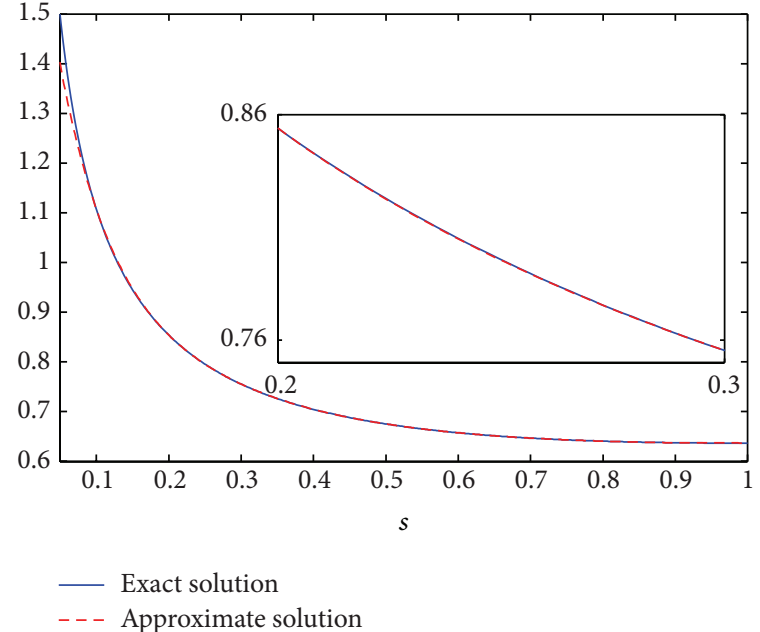

(b) $\varphi_{2}(s)$ and $\varphi_{2,5}(s)$

FIgURE 1: Comparison of the exact and approximated solutions for Example 2.

TABLE 1: Numerical results for Example 2.

\begin{tabular}{|c|c|c|c|c|c|c|}
\hline \multirow{2}{*}{$s_{i}=0.1 i$} & \multicolumn{2}{|c|}{ Exact solution } & \multicolumn{2}{|c|}{ Proposed method } & \multicolumn{2}{|c|}{ TEM } \\
\hline & $\varphi_{1}\left(s_{i}\right)$ & $\varphi_{2}\left(s_{i}\right)$ & $\varphi_{1,5}\left(s_{i}\right)$ & $\varphi_{2,5}\left(s_{i}\right)$ & $\varphi_{1,5}\left(s_{i}\right)$ & $\varphi_{2,5}\left(s_{i}\right)$ \\
\hline$i=1$ & 0.2153 & 1.1072 & 0.215290 & 1.107242 & 0.215169 & 1.107682 \\
\hline$i=2$ & 0.3259 & 0.8541 & 0.325884 & 0.854115 & 0.325780 & 0.854328 \\
\hline$i=3$ & 0.4276 & 0.7555 & 0.427565 & 0.755497 & 0.427509 & 0.755881 \\
\hline$i=4$ & 0.5293 & 0.7046 & 0.529335 & 0.704608 & 0.529611 & 0.704209 \\
\hline$i=5$ & 0.6350 & 0.6752 & 0.635031 & 0.675237 & 0.635295 & 0.675831 \\
\hline$i=6$ & 0.7470 & 0.6575 & 0.747040 & 0.657498 & 0.747548 & 0.657637 \\
\hline$i=7$ & 0.8672 & 0.6468 & 0.867187 & 0.646770 & 0.867735 & 0.646340 \\
\hline$i=8$ & 0.9971 & 0.6406 & 0.997089 & 0.640586 & 0.997818 & 0.640385 \\
\hline$i=9$ & 1.1383 & 0.6375 & 1.138298 & 0.637503 & 1.138803 & 0.637804 \\
\hline$i=10$ & 1.2924 & 0.6366 & 1.292388 & 0.636619 & 1.292765 & 0.636391 \\
\hline
\end{tabular}

Example 3. Let us consider the Abel integral equations system:

$$
\begin{aligned}
& s \varphi_{1}(s)+\sqrt{s} \varphi_{2}(s)+\varphi_{3}(s) \\
&= f_{1}(s)+\int_{0}^{s} \frac{\varphi_{1}(t)+\varphi_{2}(t)+\varphi_{3}(t)}{\sqrt{s-t}} d t, \\
& \varphi_{1}(s)-s^{2} \varphi_{2}(s)+(s+1) \varphi_{3}(s) \\
&=f_{2}(s)+\int_{0}^{s} \frac{\varphi_{1}(t)+\varphi_{2}(t)-\varphi_{3}(t)}{\sqrt{s-t}} d t, \\
&(2 s-5) \varphi_{1}(s)+\varphi_{2}(s)-\left(s^{2}-1\right) \varphi_{3}(s) \\
&=f_{3}(s)+\int_{0}^{s} \frac{\varphi_{1}(t)-\varphi_{2}(t)+\varphi_{3}(t)}{\sqrt{s-t}} d t,
\end{aligned}
$$

$$
\begin{aligned}
f_{1}(s)= & (s+1) \tan ^{-1}\left(\frac{1}{\sqrt{s}}\right)-\frac{\pi}{2}(s+1)+s(3 s+3) \\
& +\sqrt{s+1}-7 \sqrt{s}-4 \sqrt{s^{3}}-\sqrt{s^{5}}, \\
f_{2}(s)= & -(s+1) \tan ^{-1}\left(\frac{1}{\sqrt{s}}\right)+\frac{\pi}{2}(s+1)+\sqrt{(s+1)^{3}} \\
& -5 \sqrt{s}-15 s^{4}-4 \sqrt{s^{3}}-16 \sqrt{s^{5}}+3, \\
f_{3}(s)= & (s+1) \tan ^{-1}\left(\frac{1}{\sqrt{s}}\right)-\frac{\pi}{2}(s+1)+(3 s+3)(2 s-5) \\
& -\left(s^{2}-1\right) \sqrt{s+1}+15 s^{2} \\
& -7 \sqrt{s}-4 \sqrt{s^{3}}+16 \sqrt{s^{5}},
\end{aligned}
$$


TABLE 2: Numerical results for Example 3.

\begin{tabular}{lccccc}
\hline$s_{i}=0.1 i$ & Exact solution & \multicolumn{3}{c}{ Approximate solution } \\
\hline$i=1$ & $\varphi_{1}\left(s_{i}\right)$ & $\varphi_{2}\left(s_{i}\right)$ & $\varphi_{3}\left(s_{i}\right)$ & $\varphi_{1,3}\left(s_{i}\right)$ & $\varphi_{2,3}\left(s_{i}\right)$ \\
$i=2$ & 3.3000 & 0.1500 & 1.0488 & 3.3000 & 0.1500 \\
$i=3$ & 3.6000 & 0.6000 & 1.0954 & 3.6000 & 0.6000 \\
$i=4$ & 3.9000 & 1.3500 & 1.1402 & 3.9000 & 1.3500 \\
$i=5$ & 4.2000 & 2.4000 & 1.1832 & 4.2000 & 2.4000 \\
$i=6$ & 4.5000 & 3.7500 & 1.2247 & 4.5000 & 3.7500 \\
$i=7$ & 4.8000 & 5.4000 & 1.2649 & 4.8000 & 5.4000 \\
$i=8$ & 5.1000 & 7.3500 & 1.3038 & 5.1000 & 7.3500 \\
$i=9$ & 5.4000 & 9.6000 & 1.3416 & 5.4000 & 9.6000 \\
$i=10$ & 5.7000 & 12.150 & 1.3784 & 5.7000 & 1.1389 \\
\hline
\end{tabular}

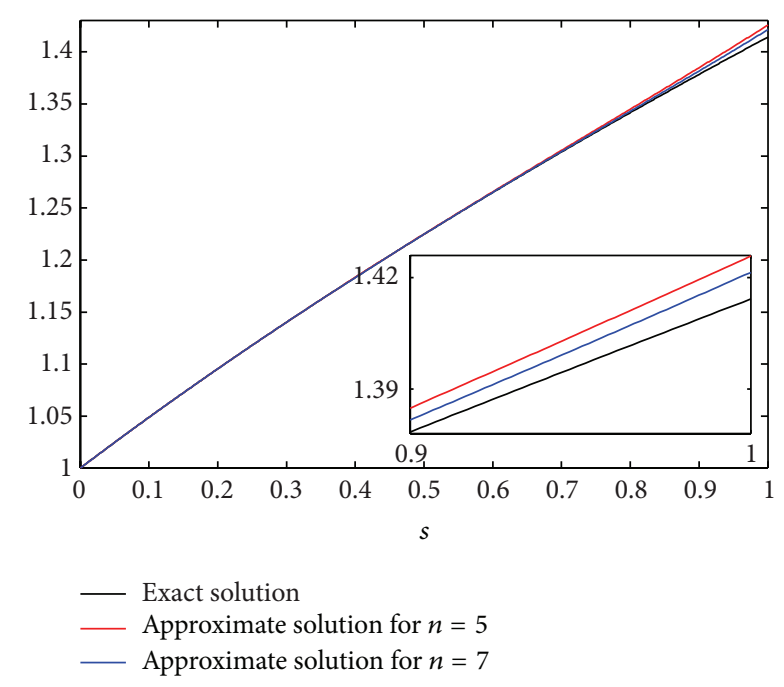

Figure $2: \varphi_{3}(s), \varphi_{3,3}(s)$, and $\varphi_{3,5}(s)$ for Example 3.

with the exact solutions $\varphi_{1}(s)=3(s+1), \varphi_{2}(s)=15 s^{2}$, and $\varphi_{3}(s)=\sqrt{s+1}$. In Table 2 , the numerical results obtained here for $n=3$ are compared with the exact solutions. It is clear that errors between the exact and approximate values of $\varphi_{1}(s)$ and $\varphi_{2}(s)$ are zero. Similarly, the series solutions of order 3 with Taylor approximation converge to the exact solutions $\varphi_{1}(s)$ and $\varphi_{2}(s)$. Also, the exact and approximate solutions of $\varphi_{3}(s)$ are compared in Figure 2 for $n=3$ and 5.

\section{Conclusions}

In this paper, we presented a useful numerical method that originated mainly from the Bernstein polynomials for solving Abel integral equations systems. This method converted the present problem to a system of linear algebraic equations for unknown functions. Having determined the unknown Bernstein coefficients of the solution functions, the series solutions were produced immediately. An interesting feature of this method is that the exact solution is derived if it is a polynomial of degree $n$ or less than $n$. On the other hand, if an exact solution is not obtainable, then the obtained series can be used for numerical purposes. In this case, more terms must be evaluated to the higher accuracy level. Additionally, the proposed technique has been compared with the Taylor expansion method. The obtained numerical results from analyzed examples illustrated that, in applications involving computations with polynomials, the Bernstein form offers efficient algorithm versus the traditional Taylor rule, for many basic functions.

\section{Conflict of Interests}

The authors declare that there is no conflict of interests regarding the publication of this paper.

\section{References}

[1] M. Khan and M. A. Gondal, "A reliable treatment of Abel's second kind singular integral equations," Applied Mathematics Letters, vol. 25, no. 11, pp. 1666-1670, 2012.

[2] L. Bougoffa, R. C. Rach, and A. Mennouni, "A convenient technique for solving linear and nonlinear Abel integral equations by the Adomian decomposition method," Applied Mathematics and Computation, vol. 218, no. 5, pp. 1785-1793, 2011.

[3] M. Abdulkawi, Z. K. Eshkuvatov, and N. M. A. Nik Long, "A note on the numerical solution of singular integral equations of cauchy type," World Academy of Science, Engineering and Technology, vol. 3, no. 9, pp. 893-896, 2009.

[4] S. Banerjea and B. Dutta, "Solution of a singular integral equation and its application to water wave problems," Journal of the Indian Institute of Science, vol. 86, no. 3, pp. 265-278, 2006.

[5] J. Helsing, "A fast and stable solver for singular integral equations on piecewise smooth curves," SIAM Journal on Scientific Computing, vol. 33, no. 1, pp. 153-174, 2011.

[6] K. Maleknejad and A. Salimi Shamloo, "Numerical solution of singular Volterra integral equations system of convolution type by using operational matrices," Applied Mathematics and Computation, vol. 195, no. 2, pp. 500-505, 2008.

[7] L. Huang, Y. Huang, and X. Li, "Approximate solution of Abel integral equation," Computers and Mathematics with Applications, vol. 56, no. 7, pp. 1748-1757, 2008.

[8] M. S. Akel and H. S. Hussein, "Numerical treatment of solving singular integral equations by using Sinc approximations," 
Applied Mathematics and Computation, vol. 218, no. 7, pp. 35653573, 2011.

[9] J. Biazar and H. Ebrahimi, "A new technique for systems of AbelVolterra integral equations," International Journal of the Physical Sciences, vol. 7, no. 1, pp. 89-99, 2012.

[10] M. H. Heydari, M. R. Hooshmandasl, F. Mohammadi, and C. Cattani, "Wavelets method for solving systems of nonlinear singular fractional Volterra integro-differential equations," Communications in Nonlinear Science and Numerical Simulation, vol. 19, no. 1, pp. 37-48, 2014.

[11] S. A. Yousefi and M. Behroozifar, "Operational matrices of Bernstein polynomials and their applications," International Journal of Systems Science, vol. 41, no. 6, pp. 709-716, 2010.

[12] M. Alipour and D. Rostamy, "Solving nonlinear fractional differential equations by Bernstein polynomials operational matrices," The Journal of Mathematics and Computer Science, vol. 5, no. 3, pp. 185-196, 2012.

[13] D. Rostamy, M. Alipour, H. Jafari, and D. Baleanu, "Solving multi-term orders fractional differential equations by operational matrices of BPs with convergence analysis," Romanian Reports in Physics, vol. 65, no. 2, pp. 334-349, 2013.

[14] D. Baleanu, M. Alipour, and H. Jafari, "The Bernstein operational matrices for solving the fractional quadratic riccati differential equations with the Riemann-Liouville derivative," Abstract and Applied Analysis, vol. 2013, Article ID 461970, 7 pages, 2013.

[15] M. Alipour and D. Baleanu, "Approximate analytical solution for nonlinear system of fractional differential equations by BPs operational matrices," Advances in Mathematical Physics, vol. 2013, Article ID 954015, 9 pages, 2013.

[16] M. Alipour, D. Rostamy, and D. Baleanu, "Solving multidimensional fractional optimal control problems with inequality constraint by Bernstein polynomials operational matrices," Journal of Vibration and Control, vol. 19, no. 16, pp. 2523-2540, 2013.

[17] M. Alipour and D. Rostamy, "BPs operational matrices for solving time varying fractional optimal control problems," The Journal of Mathematics and Computer Science, vol. 6, pp. 292304, 2013.

[18] A. Jafarian, S. Measoomy Nia, A. K. Golmankhaneh, and D. Baleanu, "Numerical solution of linear integral equations system using the Bernstein collocation method," Advances in Difference Equations, vol. 2013, article 123, 2013.

[19] V. K. Singh, R. K. Pandey, and O. P. Singh, "New stable numerical solutions of singular integral equations of Abel type by using normalized Bernstein polynomials," Applied Mathematical Sciences, vol. 3, no. 5, pp. 241-255, 2009.

[20] O. P. Singh, V. K. Singh, and R. K. Pandey, "A stable numerical inversion of Abel's integral equation using almost Bernstein operational matrix," Journal of Quantitative Spectroscopy and Radiative Transfer, vol. 111, no. 1, pp. 245-252, 2010.

[21] S. Dixit, O. P. Singh, and S. Kumar, "A stable numerical inversion of generalized Abels integral equation," Applied Numerical Mathematics, vol. 62, no. 5, pp. 567-579, 2012.

[22] O. R. Işik, M. Sezer, and Z. Güney, "Bernstein series solution of a class of linear integro-differential equations with weakly singular kernel," Applied Mathematics and Computation, vol. 217, no. 16, pp. 7009-7020, 2011.

[23] A. M. Wazwaz, Linear and Nonlinear Integral Equations: Methods and Applications, Higher Education Press, Beijing, China; Springer, Berlin, Germany, 2011.
[24] B. N. Mandal and S. Bhattacharya, "Numerical solution of some classes of integral equations using Bernstein polynomials," Applied Mathematics and Computation, vol. 190, no. 2, pp. 17071716, 2007. 


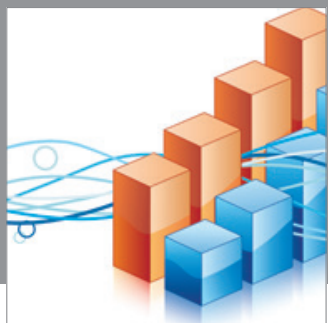

Advances in

Operations Research

mansans

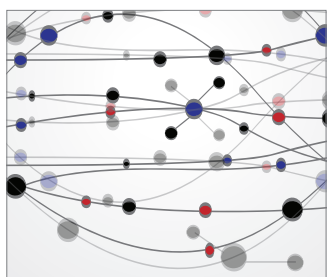

The Scientific World Journal
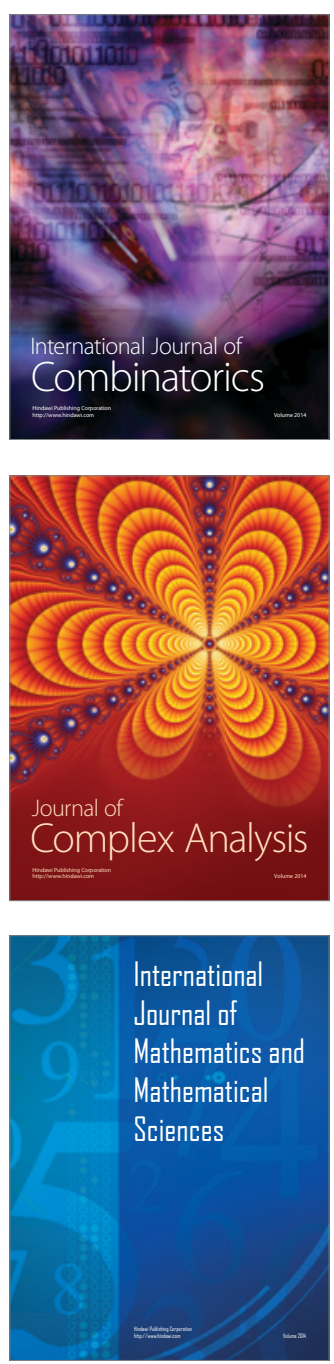
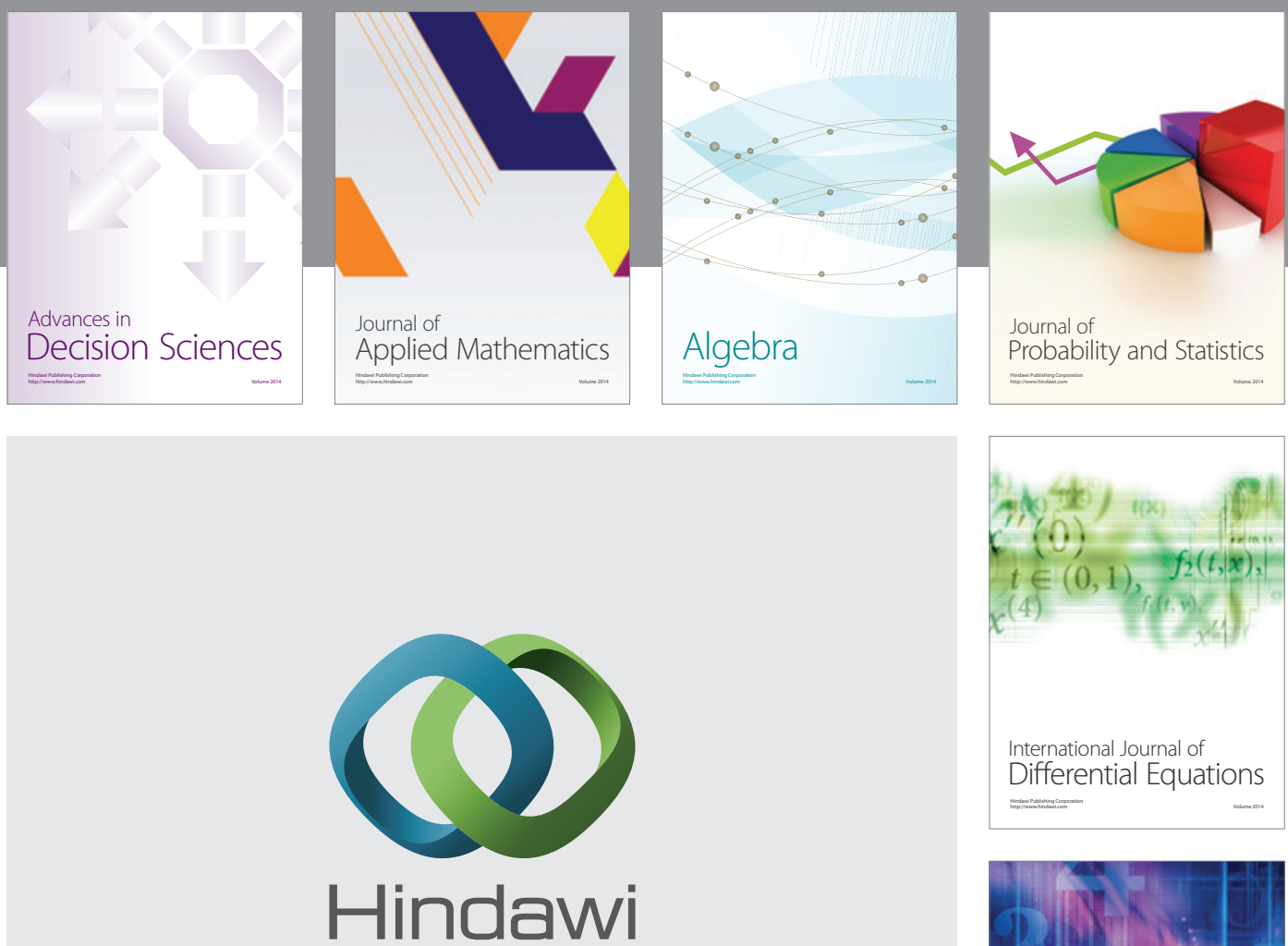

Submit your manuscripts at http://www.hindawi.com
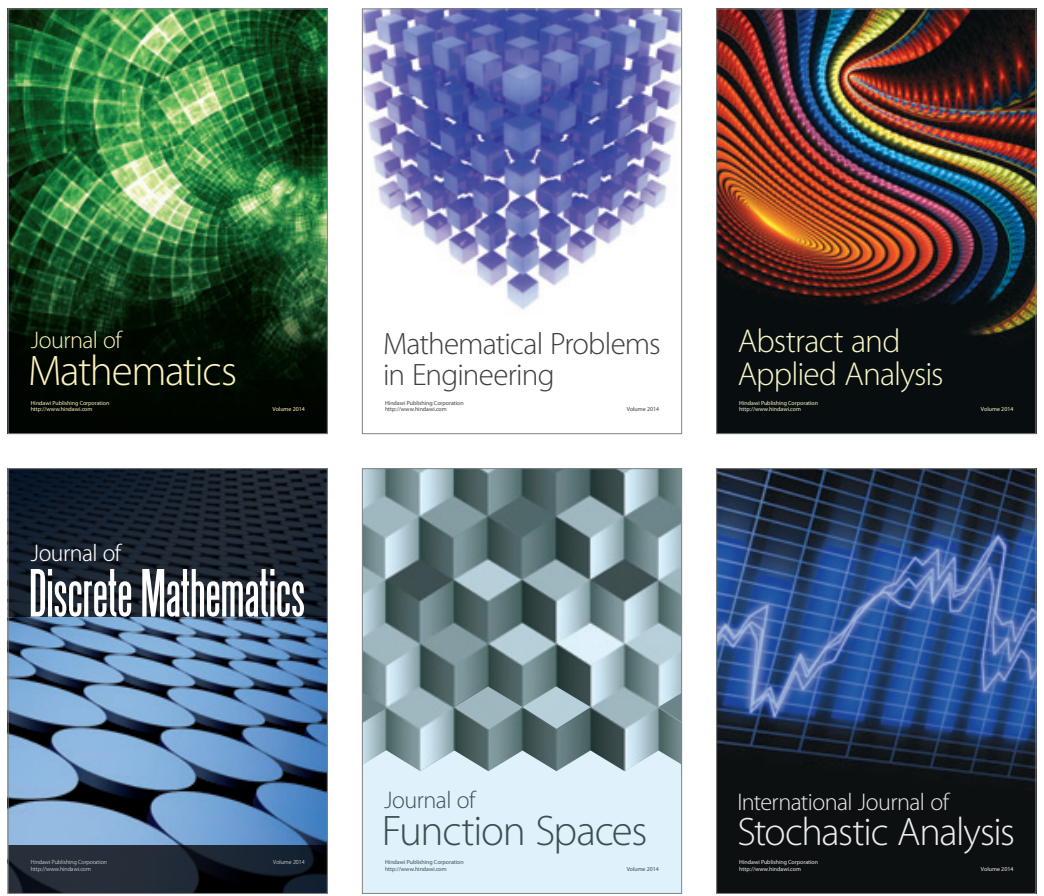

Journal of

Function Spaces

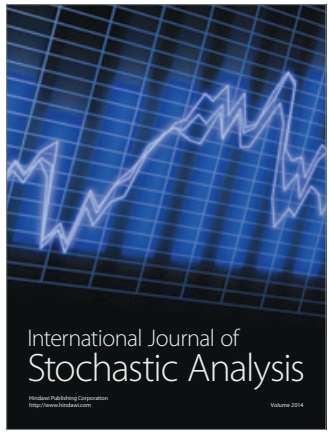

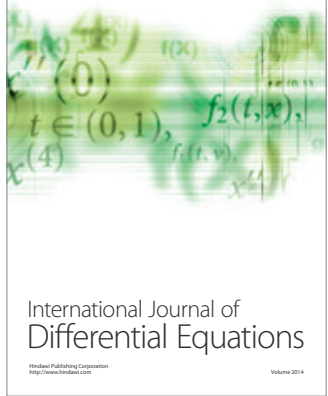
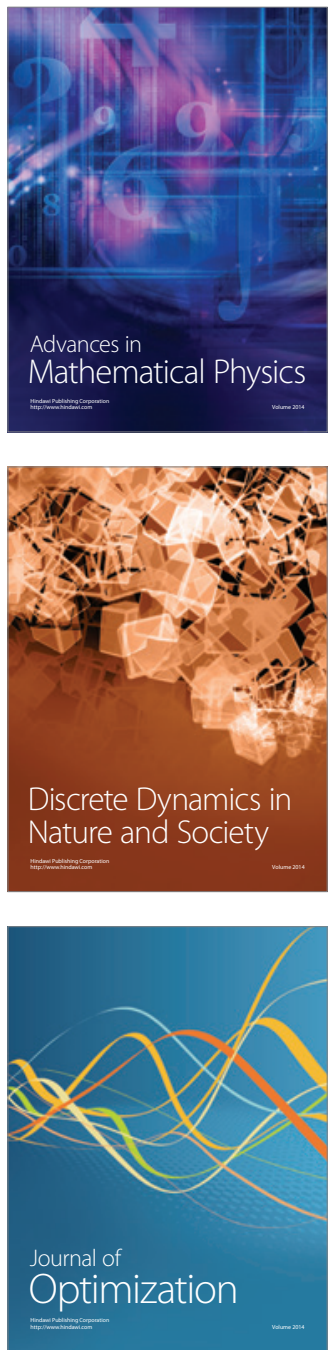